\title{
RESULTADOS DE UN PROGRAMA DE OXÍGENOTERAPIA HIPERBÁRICA PARA EL TRATAMIENTO DE ÚLCERAS ARTERIALES
}

\author{
EDUARdo DuRán PINILLA \\ ALEJANDRo Buitrago SALINAS ${ }^{2}$ \\ GERMÁN BAQUERO SASTRE ${ }^{3}$ \\ ${ }^{1}$ Médico Neurocirujano - Director de la Unidad de Medicina Hiperbárica del Hospital Militar Central. \\ ${ }^{2}$ Médico General - Unidad de Medicina Hiperbárica del Hospital Militar Central. \\ ${ }^{3}$ Fisioterapeuta - Epidemiólogo. Asesor de Investigaciones de la Unidad de Medicina Hiperbárica \\ del Hospital Militar Central
}

Recibido: Julio 23 de 2012 Aceptado: Noviembre 06 de 2012

\section{Resumen}

La Enfermedad Arterial Oclusiva Crónica (EAOC), es una entidad de sensibles impactos, dado que en sus mecanismos fisiopatológicos se genera una isquemia e hipoxia, que desvitalizan elementos tisulares dando paso a alteraciones integumentarias con aparición de úlceras de difícil manejo que se pueden sobreinfectar e incluso generar la amputación de extremidades. No siendo frecuente que en los tratamientos clínicos se dé la aplicación de oxígenoterapia hiperbárica, que podría reversar los mecanismos de alteraciones fisiopatológicas. Este trabajo corresponde a un estudio transversal desarrollado en la Unidad de Medicina Hiperbárica del Hospital Militar Central. Que contó con cuarenta y cinco pacientes intervenidos entre los años de 2004 a 2008.que presentaron úlceras arteriales arteriales. Los pacientes tenían una edad media de 68 años, habiendo recibido el 50\% de ellos, un número de 20 sesiones, con una presión de 1.5 atmosferas en el $50 \%$ de los casos, lográndose una satisfactoria evolución de sus compromisos en el $77.7 \%$ de los casos ( $n=35$, error estándar $=0.03$ ).

Palabras Claves: Enfermedad arterial, úlceras arteriales, oxígenoterapia hiperbárica, oxígenoterapia hiperbárica en úlceras arteriales, tratamiento de úlceras arteriales.

\section{RESULTS OF A PROGRAM OF HYPERBARIC OXYGEN THERAPY FOR THE TREATMENT OF ARTERIAL ULCERS}

\begin{abstract}
Chronic Occlusive Arterial Disease (COAD) is an entity that has a significant impact since over its pathophysiological mechanisms ischemia and hypoxia are generated thus devitalizing tissue elements and giving rise to disturbances with the appearance of integumentary ulcers that can be unmanageable and even lead to superinfection and subsequent amputation of limbs. However, it is not common in clinical treatment to administer hyperbaric oxygen therapy even though it might reverse the mechanisms
\end{abstract}

* Correspondencia: Dr. Eduardo Durán Pinilla, Transversal 3 A Número 49-00 Tercer piso Central. Teléfono (57-1) 3486868 Extensión 5287. Dr. Alejandro Buitrago Salinas. Transversal 3 A Número 49 - 00 Tercer piso Central, Teléfono (57-1) 3486868, Extensión 5287, FT MSC Germán Baquero Sastre. Transversal 3 A Número 49 - 00 Tercer piso Central, Teléfono (57 -1) 3486868, Extensión 5287 
of such pathophysiological disturbances. This work corresponds to a cross-sectional study carried out in the Hyperbaric Medicine Unit of the Central Military Hospital in 45 patients with mean age of 68 years who had arterial ulcers and underwent surgical procedures between 2004 and 2008. Fifty percent of patients received 20 sessions with a pressure of 1.5 atmospheres in $50 \%$ of cases, achieving a satisfactory evolution of the involved areas in $77.7 \%$ of cases $(n=35$, standard error $=0.03$ )

Keywords: Arterial disease, arterial ulcers, hyperbaric oxygen therapy, hyperbaric oxygen in arterial ulcers, arterial ulcers treatment.

\title{
RESULTADOS DE UM PROGRAMA DE OXIGENOTERAPIA HIPERBÁRICA PARA O TRATAMENTO DE ÚLCERAS ARTERIAIS
}

\begin{abstract}
Resumo
A doença arterial obstrutiva crônica (DAOC) é uma entidade de sensíveis impactos, dado que em seus mecanismos fisiopatológicos se gera uma isquemia e hipoxia, que desvitalizam elementos tisulares levando a alterações tegumentárias com aparição de úlceras de difícil tratamento, que podem se infetar $e$ inclusive gerar a amputação de membros. Não sendo frequente que nos tratamentos clínicos se dê a aplicação de oxigenoterapia hiperbárica, que poderia reverter os mecanismos de alterações fisiopatológicas. Este trabalho corresponde a um estudo transversal desenvolvido na Unidade de Medicina Hiperbárica do Hospital Militar Central, que contou com quarenta e cinco pacientes intervindos entre os anos de 2004 a 2008, que apresentaram úlceras arteriais. Os pacientes tinham uma idade média de 68 anos, havendo recebido $50 \%$ deles, um número de 20 sessões, com una pressão de 1.5 atmosferas em $50 \%$ dos casos e conseguindo-se uma satisfatória evolução de seus compromissos em $77.7 \%$ dos casos $(n=35$, erro padrão $=0.03$ ).
\end{abstract}

Palavras chave: Doença arterial, úlceras arteriais, oxigenoterapia hiperbárica, oxigenoterapia hiperbárica em úlceras arteriais, tratamento de úlceras arteriais.

\section{Introducción}

La Enfermedad Arterial Oclusiva Crónica (EAOC) representa una entidad, dentro de la cual, sus mecanismos fisiopatológicos contemplan la alteración en la luz de una estructura vascular arterial por daño de la misma, comprometiéndose la perfusión de los tejidos irrigados por el vaso y generándose una isquemia en ellos. Ésto da lugar a una hipoxia tisular, al punto de llegar a una desvitalización del tejido, que favorece metabolismos y detritos anaeróbicos, que facilitaran la generación de infecciones por las cuales se tendrá que llevar a la práctica de amputaciones de las extremidades comprometidas(1-3).

Con la presentación de la EAOC y sus cambios fisiopatológicos en la perfusión vascular, uno de los tejidos que sufre más afectaciones al disminuirse el aporte de nutrientes, para mantener sus propiedades tróficas y mecánicas es el integumentario. En él se produce la aparición de úlceras arteriales, las cuales con su manifestación además de afectar la integridad epitelial, favorecen situaciones de sobreinfección que podrían terminar llevando a la práctica de amputaciones en el paciente. Procedimiento con sensibles impactos psicosociales y económicos por las afecciones en el proceso de vida del paciente, desempeños funcionales, laborales y costos del proceso de rehabilitación para un uso protésico (1-3).

Las causas más frecuentes para el desencadenamiento de la EAOC están relacionadas con la arterioesclerosis, la hipertensión arterial, hipercolesterolemia, hábito tabáquico y la diabetes Mellitus. Un 20\% de las personas con la afección fallecen en el primer año de evolución de la enfermedad $(1,4)$.

Las formas más comúnmente empleadas para el tratamiento de la insuficiencia arterial, comprenden medidas 
farmacológicas, y procesos quirúrgicos, y cuando se han establecido las úlceras se deberán practicar frecuentes curaciones y lavados para evitar procesos infecciosos. Las infecciones afectan el tiempo y las posibilidades de cicatrización, que pueden tardar un promedio de seis meses, repercutiendo en los desempeños funcionales y el estilo de vida de la persona (4, 7-10).

La oxígenoterapia hiperbárica comprende la administración de oxígeno al $100 \%$ a una presión mayor que la atmosférica, presiones elevadas y controladas, que incrementa las concentraciones del gas en el organismo generando dentro de sus efectos un control a la presentación de procesos de edema. Al contrarrestar los productos de metabolismos anaeróbicos, se potencializa la acción bactericida de macrófagos, como de las células de defensa, y se favorecen procesos de angiogénesis, a partir de ellos, en la EAOC, se tendrían factores que ante la presencia de úlceras ayudarían a la revitalización del tejido, la recuperación de su nutrición, y la promoción de adecuados procesos de cicatrización. Éstos llevarían al cierre de la úlcera, reduciendo los tiempos del proceso y haciendo que se dé una recuperación con mejores posibilidades. La ausencia de complicaciones, acelera el retorno a las acciones funcionales y el retorno a la cotidianidad del paciente, con sus consecuentes ventajas $(1,11-14)$.

Hasta el momento, a pesar de tenerse elementos fisiológicos por los cuales la oxígenoterapia hiperbárica sería un elemento fundamental para el tratamiento de la EAOC y la presencia dentro de ella de úlceras, no es una de las primeras opciones para el manejo de los pacientes que presentan este cuadro.

\section{Metodología}

En este trabajo y con el propósito de describir los resultados de la aplicación del oxígeno hiperbárico en el tratamiento de úlceras en la EAOC, se desarrolló un estudio no experimental, observacional, de corte transversal $(15,16)$.

El estudio se adelantó con pacientes remitidos para intervención a la Unidad de Medicina Hiperbárica del Hospital Militar Central, con presencia de úlceras arteriales en el periodo de tiempo comprendido entre los años de 2004 a 2008.

Los criterios de inclusión, que se tuvieron en cuenta para las personas que formaron parte de la población de estudio comprendieron; que se presentara una úlcera arterial, y se hubiera remitido para manejo en la Unidad de Medicina Hiperbárica del Hospital Militar Central dentro del periodo de tiempo de los años 2004 a 2008. No se consideraron como factores que impidieran el hecho de entrar a ser parte del estudio, el tiempo de evolución de la EAOC, o de la presentación de la úlcera, y los tratamientos anteriores a los que hubieran podido ser sometidos médicamente los pacientes.

Como criterios de exclusión para que un paciente con úlcera arterial no formara parte de la población de estudio, se definieron; el que tuviera concomitantemente enfermedades oncológicas, situaciones de inmunosupresión, o enfermedades degenerativas, y que hubiera sido intervenido con oxígenoterapia hiperbárica antes del año 2004 o con posterioridad al año 2008.

Todos los pacientes que cumpliendo los criterios de inclusión entraron a hacer parte de la población de estudio, dieron su consentimiento informado de manera verbal y por escrito para ser intervenidos con oxígenoterapia en búsqueda de tratar sus úlceras arteriales.

La manera de selección de la población, correspondió a la realización de un censo de todas las personas con EAOC y presencia de úlceras a causa de ella, que fueron remitidas para manejo en la Unidad de Medicina Hiperbárica del Hospital Militar Central entre el año de 2004 y el de 2008.

En los procedimientos aplicados en el estudio los pacientes al ingreso a la Unidad de Medicina Hiperbárica fueron valorados médicamente, configurado su anamnesis, ubicación de la úlcera y especificación de sus características clínicas de presentación.

Posterior a la valoración los pacientes fueron intervenidos con la aplicación de oxígeno hiperbárico en cámaras monoplaza de manera diaria, por espacio de una hora.

Previo al inicio del esquema de intervención, se desarrollo en los miembros de la población de estudio, un numero de tres sesiones consideradas de adaptación, donde se miraba la respuesta psicológica de la persona al permanecer en la cámara de oxígeno hiperbárico, adaptación al ambiente de la misma y su tolerancia a la administración del tratamiento. De tal manera, que si no se encontraban reacciones de claustrofobia o de intolerancia al procedimiento por razones fisiológicas, 
el paciente iniciaba el esquema de intervención con aumento de presión.

Al final del programa de intervención, los pacientes fueron nuevamente valorados medicamente, mirando el grado de cierre de la úlcera tratada, y los cambios registrados en sus características clínicas, en comparación a las establecidas en la valoración inicial.

Para los procesos de análisis de resultados, se calcularon medidas estadísticas descriptivas de resumen y de dispersión, prevalencias crudas y especificas con sus correspondientes niveles de error estándar. Y para encontrar las relaciones del género, la edad, el numero de sesiones, y las atmósferas empleadas en el tratamiento de oxígenoterapia hiperbárica, con la mejoría de la úlcera arterial se realizaron pruebas de chi cuadrada con un grado de libertad y una $\mathrm{p}<\mathrm{de} 0.05$.

\section{Resultados}

En la realización del estudio participaron un total de 45 personas, quienes fueron intervenidas en la Unidad de Medicina Hiperbárica del Hospital Militar Central en el periodo de tiempo de los años de 2004 a 2008 , por casos de úlceras arteriales, de las cuales el $53.33 \%(n=24$, error estándar $=0.06)$ eran de género masculino (Tabla 1 ).

La media de edad de la población de estudio fue de 67.5 años con una desviación estándar de 8.5 años, el $25 \%$ de la población se encontró por debajo de los 63 años, y el $75 \%$ por debajo de los 73 años, el 50\% de las personas participantes en el estudio tenían una edad de 68 años (Tabla 1).

El promedio de sesiones de oxígenoterapia hiperbárica, para tratar las úlceras arteriales que predominaron con carácter exclusivo en miembros inferiores en la mayoría de los casos relacionadas con enfermedad diabética, fue de 26 sesiones. El $50 \%$ de las personas recibió un número de veinte sesiones y el $75 \%$ de las personas intervenidas recibió menos de treinta y cuatro sesiones de tratamiento (Tabla 1).

Dentro de la intervención cumplida, la media de atmósferas de presión (ATA) que se aplicaron dentro del manejo de oxígenoterapia hiperbárica a personas con úlceras arteriales fue de 1.69 ATA, con una desviación estándar de 0.45 ATA. El $50 \%$ de la población recibió 1.5 ATA, y $75 \%$ de los miembros formantes de ella recibieron menos de 1.5 ATA (Tabla 1).
Un $77.7 \%$ ( $n=35$, error estándar $=0.03$ ) de las personas de la población de estudio tuvieron resultados satisfactorios, con el esquema de manejo de oxígenoterapia hiperbárica, representados en el cierre de la úlcera arterial inicialmente evidenciada. El $15.5 \%(n=7$, error estándar $=0.13$ ) de las personas no presentaron mejorías en sus úlceras arteriales, permaneciendo éstas en las mismas condiciones detectadas en la valoración clínica inicial (Tabla 1) (Figura 1, Figura 2 y Figura 3). $\mathrm{El} 85.71 \%(\mathrm{n}=18$, error estándar $=0.03)$ de las personas de género femenino, que hicieron parte de la población de estudio, registraron una mejoría con el cierre de la úlcera arterial, luego de haber participado del esquema de tratamiento con la administración de oxígeno hiperbárico. Mientras que estas mismas circunstancias se dio mejoría en el $70.83 \%$ de la personas de género masculino $(n=17$, error estándar $=0.05)(X 2=1.43, p$ $>$ de 0.05). Con lo cual, en las medidas de asociación se concretó que las mujeres tenían 2.47 veces más posibilidades de tener resultados satisfactorios con el cierre de la úlcera arterial, al ser manejadas con oxígenoterapia hiperbárica, respecto al género masculino (Intervalo de Confianza al 95\% 0.56 - 10.69) (Tabla 2).

El 87.5\% ( $n=14$, error estándar $=0.03)$ de las personas de la población de estudio, que fueron intervenidas con más de veinte sesiones de oxígenoterapia hiperbárica, lograron al culminar el programa de intervención, significativos resultados en el tratamiento de las úlceras arteriales, mientras que para el caso de quienes recibieron menos de veinte sesiones de manejo el $74.07 \%$ $(n=20$, error estándar $=0.05)$ lograron los mismos resultados en cuanto a la resolución de su úlcera arterial (X2 $=1.09, \mathrm{p}>$ de 0.05) (Tabla 2).

Las personas, que recibieron más de veinte sesiones de tratamiento para el cierre de úlceras arteriales con manejo de oxígenoterapia hiperbárica, tuvieron 2.45 veces más probabilidades de llegar a tener el cierre de la úlcera en comparación con aquellas personas que recibieron menos de veinte sesiones para el manejo de sus úlceras arteriales (Intervalo de Confianza al 95\% 0.44 - 13.32) (Tabla 2).

Finalmente, las personas miembros de la población de estudio que recibieron más 1.5 ATA en la administración del oxígeno hiperbárico, un $88.8 \%(n=8$, error estándar $=0.03$ ) de ellas, alcanzó en los resultados del manejo, a manifestar el cierre de la úlcera, mientras que en quienes recibieron menos de 1.5 ATA en el tratamiento un $76.47 \%(n=26$, error estándar $=0.03)$, 
Durán E., Buitrago A., BaQuero G.

Tabla 1. Características de la población y el tratamiento con oxígeno hiperbárico para el tratamiento de úlceras arteriales.

\begin{tabular}{|c|c|c|c|c|c|c|}
\hline CARACTERÍSTICA & PORCENTAJE & MEDIA & $\begin{array}{l}\text { DESVIACIÓN } \\
\text { ESTÁNDAR }\end{array}$ & $\begin{array}{c}\text { PRIMER } \\
\text { CUARTIL } \\
25 \% \\
\end{array}$ & $\begin{array}{c}\text { SEGUNDO } \\
\text { CUARTIL } \\
50 \% \\
\end{array}$ & $\begin{array}{c}\text { TERCER } \\
\text { CUARTIL } \\
\mathbf{7 5 \%} \\
\end{array}$ \\
\hline $\begin{array}{l}\text { Género } \\
\text { Masculino } \\
\text { Femenino }\end{array}$ & $\begin{array}{c}53.33 \% \\
(\mathrm{n}=24 \\
* \mathrm{E}=0.06) \\
46.66 \% \\
(\mathrm{n}=21, \\
* \mathrm{E}=0.07)\end{array}$ & $* * \mathrm{NP}$ & $* * \mathrm{NP}$ & $* * \mathrm{NP}$ & $* * \mathrm{NP}$ & $* * \mathrm{NP}$ \\
\hline Edad & NP & 67.5 años & 8.5 años & 63 años & 68 años & 73 años \\
\hline Número de Sesiones & NP & 26 sesiones & 18.8 sesiones & 15 sesiones & 20 sesiones & 34 sesiones \\
\hline Presión - ATA & NP & 1.69 ATA & 0.45 ATA & $1.5 \mathrm{ATA}$ & $1.5 \mathrm{ATA}$ & $1.5 \mathrm{ATA}$ \\
\hline $\begin{array}{l}\text { Mejoría } \\
\text { Total } \\
\text { Parcial } \\
\text { Sin Mejoría }\end{array}$ & $\begin{array}{c}77.7 \% \\
(\mathrm{n}=35 \\
\text { *E.E=0.03) } \\
6.6 \% \\
(\mathrm{n}=3, \\
\text { *E. } \mathrm{E}=0.13) \\
15.5 \% \\
(\mathrm{n}=7, \\
\text { *E. } \mathrm{E}=0.13)\end{array}$ & $* * N P$ & $* * \mathrm{NP}$ & $* * \mathrm{NP}$ & $* * \mathrm{NP}$ & $* * \mathrm{NP}$ \\
\hline
\end{tabular}

* E.E $=$ Error Estándar

** NP= Cálculo No Posible

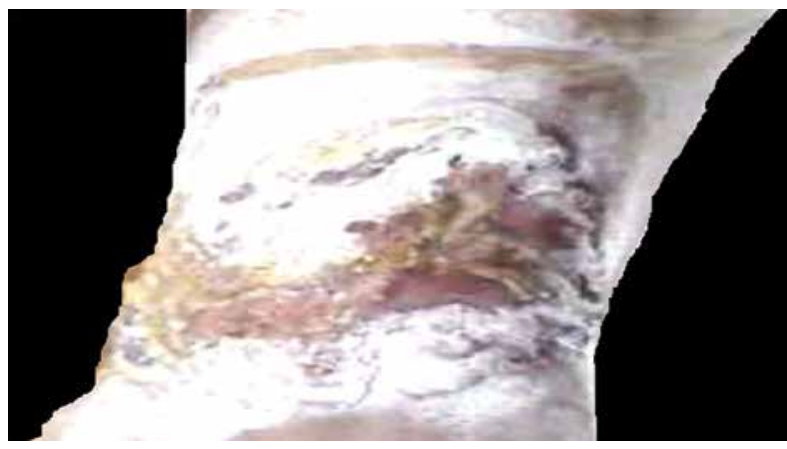

Figura 1. Vista anterior de una úlcera arterial en miembro inferior al ingreso, donde se observa escaso tejido de granulación en un paciente antes de ser intervenido con oxígeno hiperbárico.

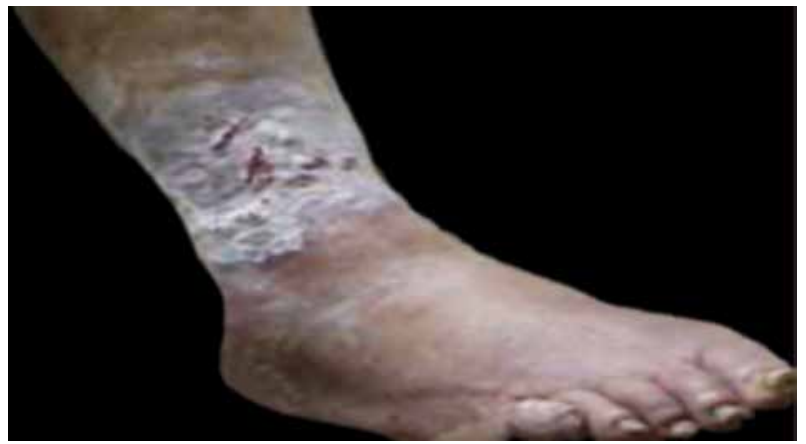

Figura 2. Evolución de la úlcera arterial en vista anterior luego de haber recibido 12 sesiones de intervención con oxígenoterapia hiperbárica.

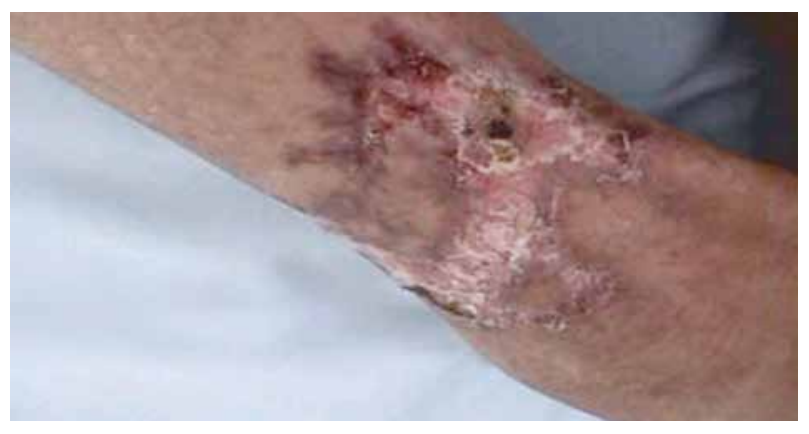

Figura 3. Estado de evolución de la úlcera arterial en vista anterior luego de haber recibido 30 sesiones de oxígeno hiperbárico. 
registraron la misma situación de evolución $(\mathrm{X} 2=0.66$ $\mathrm{p}>\mathrm{de}$ 0.05). Por lo tanto, las personas que recibieron más de 1.5 ATA en el manejo de oxígeno hiperbárico, tuvieron 2.46 veces más probabilidades de tener resultados satisfactorios en el tratamiento de sus úlceras arteriales, con el cierre de las mismas con relación a quienes recibieron menos de 1.5 ATA (Intervalo de Confianza al 95\% 0.27 - 21.97) (Tabla 2).

\section{Discusión}

La EAOC es una entidad que en la actualidad viene observando tendencias crecientes en su frecuencia de presentación dentro de la población. Si se tiene en cuenta que entre sus factores desencadenantes principales se encontrarían la enfermedad hipertensiva arterial y trastornos metabólicos como la diabetes, que en la actualidad tienen importantes cifras de personas afectadas, razón por la cual las úlceras arteriales como complicación de esta entidad, también tienden a aumentar su ocurrencia entre los afectados (1-6).

La presencia de úlceras arteriales es una complicación que genera grandes impactos, pues al tenerse la solución del tejido epitelial como barrera sistémica de protección, la primera situación de preocupación será el desencadenamiento de infecciones por contacto con el medio y situaciones de inmunocompetencia del paciente, que pueden favorecer este hecho. Lo anterior, clínicamente dificultará los manejos terapéuticos y hará que se retarde el cierre del compromiso intertegumentario, dado que con la lesión en la integridad epitelial se dan fenómenos inflamatorios tisulares que con la infección y detritos anaeróbicos se incrementaran, haciendo que se dificulten los procesos de regeneración y llevando a que la presencia de la úlcera continúe $(1,11,17)$.

La ocurrencia de las úlceras arteriales al darse preponderantemente en miembros inferiores, impiden las posibilidades de apoyo de la extremidad, alterando los procesos funcionales de marcha y desplazamiento. Por esta razón a los deletéreos efectos y riesgos clínicos que debe tener el paciente, se sumarán los de orden psicosocial, causados por la pérdida de contactos sociales y laborales, que se suman a los impactos económicos del tratamiento y costos de incapacidades, reducción de la productividad y preponderantemente de los niveles de calidad de vida de la persona, interfiriendo en las posibilidades de lograr satisfacción y bienestar según sus intereses y expectativas (1-3).

Tabla 2. Relaciones de ciertos factores con la mejoría lograda en personas con úlceras arteriales al ser tratadas con oxígeno hiperbárico.

\begin{tabular}{|c|c|c|c|c|c|}
\hline FACTOR & $\begin{array}{l}\text { MEJORÍA } \\
\text { TOTAL }\end{array}$ & $\begin{array}{l}\text { MEJORIA PARCIAL } \\
\text { O SIN MEJORIA }\end{array}$ & $\begin{array}{l}\text { VALOR } \\
\text { X2 }\end{array}$ & $\begin{array}{l}\text { RAZÓN DE } \\
\text { MOMIOS }\end{array}$ & $\begin{array}{l}\text { INTERVALO DE } \\
\text { CONFIANZA 95\% }\end{array}$ \\
\hline $\begin{array}{l}\text { Femenino } \\
\text { Masculinero }\end{array}$ & 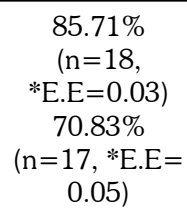 & $\begin{array}{c}14.29 \% \\
(n=3, * E . E=0.22) \\
29.17 \% \\
(n=7, * E . E=0.14)\end{array}$ & $\begin{array}{c}1.43 \\
P>0.05\end{array}$ & 2.47 & $0.56-10.69$ \\
\hline $\begin{array}{l}\text { Número Sesiones } \\
\text { Más de } 20 \text { Sesiones } \\
\text { Menos de } 20 \text { Sesiones }\end{array}$ & $\begin{array}{c}87.5 \% \\
(n=14 \\
* E . E=0.03) \\
74.07 \% \\
(n=20 \\
* E . E=0.05)\end{array}$ & $\begin{array}{c}12.5 \% \\
(n=2, E . E=0.29) \\
25.93 \% \\
(n=7, * E . E=0.15)\end{array}$ & $\begin{array}{c}1.09 \\
P>0.05\end{array}$ & 2.45 & $0.44-13.32$ \\
\hline $\begin{array}{l}\text { Presiones Atmosféricas - ATA } \\
\text { Más de 1.5 ATA } \\
\text { Menos de 1.5 ATA }\end{array}$ & $\begin{array}{c}88.8 \% \\
(\mathrm{n}=8, \\
* E . E=0.03) \\
76.47 \% \\
(\mathrm{n}=26 \\
* E: E=0.04)\end{array}$ & $\begin{array}{c}11.2 \% \\
\left(n=1,{ }^{*} E . E=* * N P\right) \\
23.53 \% \\
(n=8, * E . E=0.12)\end{array}$ & $\begin{array}{c}0.66 \\
P>0.05\end{array}$ & 2.46 & $0.27-21.97$ \\
\hline
\end{tabular}

* E.E $=$ Error Estándar

** NP $=$ Calculo No Posible 


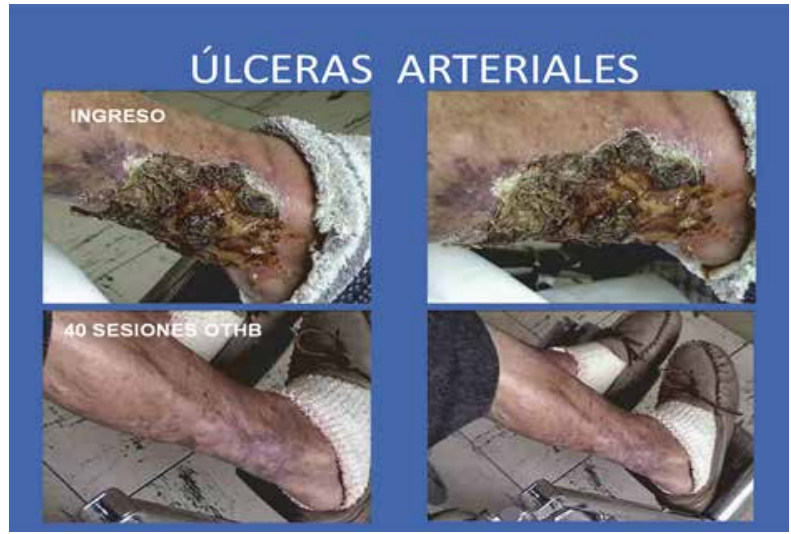

Figura 4. Estado de evolución de una úlcera arterial en cara posterior de piernas, donde se observa al ingreso unas lesiones costrificadas, necróticas y sobreinfectadas y después de 40 sesiones de OHB, se observa completa mejoría.

Con la presencia de la infección de las úlceras arteriales se genera el riesgo, de que ésta pueda diseminarse por vía hematógena, generando síndromes compartiméntales que reduzcan más la perfusión vascular tisular y causen riesgos de gangrena. Por lo consiguiente, ante respuestas negativas a las medidas farmacológicas de cobertura con antibioticoterapia, se debe llevar a la persona a la práctica de amputaciones que generaran costos psicosociales y económicos por el posterior proceso de rehabilitación al uso protésico $(18,19)$.

Las posibilidades de infección de las úlceras arteriales, no solamente son altas por contacto con organismos del medio, sino también por el hecho de que en casos de diabetes el paciente pierde mecanismos de inmunocompetencia, dando una interacción que dificultará el cierre del compromiso (18).

Los tratamientos que se instauran ante las úlceras arteriales, lavados y curaciones requieren de largos periodos y un adecuado manejo que se puede extender incluso por periodos mayores a 6 meses, luego de los cuales la eficacia se pierde en la resolución del problema, pasándose a requerir la práctica de amputaciones para preservar la vitalidad de los segmentos proximales del miembro y la sobrevida del paciente $(4,7-10,18,19)$

La oxígenoterapia hiperbárica sería una verdadera alternativa de tratamiento, con altas probabilidades de eficacia, si se tiene en cuenta que la alta exposición a

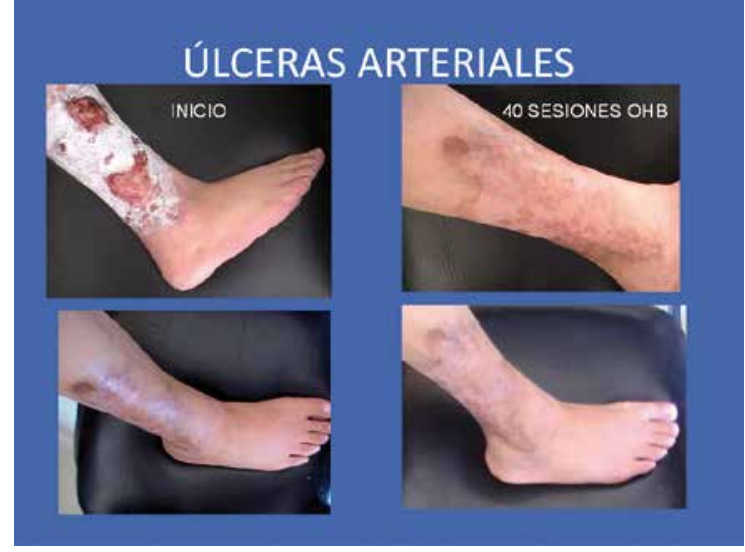

Figura 5. Estado de evolución de una úlcera arterial en cara lateral de piernas, de varios años de evolución, en donde se observa al ingreso unas lesiones profundas con escaso tejido de granulación, y después de 40 sesiones de OTHB, se observa completa mejoría.

oxígeno con presiones controladas, lograría reducir en primera instancia, los procesos inflamatorios tisulares por mecanismos de vasoconstricción, rompiendo la cascada del proceso inflamatorio. Entonces, se reducirían los riesgos de complicaciones referidas como el síndrome compartimental, además con las altas concentraciones de oxígeno se favorecen los mecanismos de oxidación celular, y se reducen los metabolismos anaeróbicos, disminuyendo la presencia de detritos que son factores favorecedores de infección $(1,11-14,17)$.

Con la aplicación de la oxígenoterapia hiperbárica, no solamente se estaría facilitando el cierre de las úlceras arteriales por control de la cascada inflamatoria, y reducción del metabolismo anaeróbico que controlaría la existencia y propagación de la infección. Sino que además, ayudaría a mecanismos de angiogénesis, que son vitales para favorecer los procesos de reparación del tejido intertegumentario, con una adecuada respuesta celular de macrófagos y fibroblastos para la producción de colágeno, ayudando a que las propiedades tróficas y mecánicas de la piel en la cicatrización sean mejores y promuevan el cierre de la úlcera $(1,11-14,17)$

Dentro de los resultados de nuestro estudio, debe tenerse en cuenta, que la media de edad de 67.5 años en la población de estudio, determina que las personas afectadas por úlceras arteriales no solo presentaban inflamación tisular e infección para demorar el cierre de la lesión; sino que este hecho también estaría re- 
ducido, si se tiene en cuenta que el proceso fisiológico de envejecimiento hace que a nivel intertegumentarios se pierdan propiedades mecánicas y tróficas de la piel. Siendo ésta menos elástica y mas reseca, lo que la hace propensa a pérdidas de su integridad, y en las situaciones sistémicas hace que los adultos mayores tengan menos inmuno-competencia, facilitando en ellos la infección. Por lo que se tendrían situaciones de interacción en el hecho de ser adulto mayor y diabético, para que no se dé el cierre de la úlcera.

Si se ven los resultados de mejoría logrados en este estudio donde un $77.7 \%(n=35$, error estándar $=0.03)$ de los miembros de estudio lograron el cierre de su úlcera, en tiempos que no fueron superiores a 34 sesiones en el $75 \%$ de los intervenidos. Podemos observar, que los resultados de la oxígenoterapia hiperbárica son satisfactorios en tiempos cortos, que no serian mayores de un mes, lo que es una mejoría significativa al promedio de seis meses, cuando solamente se da la práctica de lavados y curaciones más el suministro de elementos de antibioticoterapia (4, 7-10).

Por otra parte, los resultados de la oxígenoterapia hiperbárica en el cierre de úlceras arteriales, no solamente serian satisfactorios al contrarrestar cascadas inflamatorias, procesos anaeróbicos, infecciosos y ayudar a las respuestas celulares para que se dé la cicatrización y la regeneración epitelial, sino que debe anotarse que también es seguro, siendo mínimos los riesgos de complicaciones o efectos secundarios que deben considerarse con la antibioticoterapia prolongada, ya que no solo se presenta la situación de resistencia a los antibióticos, que por lo general son de importante nivel de generación, sino que algunos de ellos reducirán niveles de hematocrito y hemoglobina $(1,11-14)$.

Resulta de interés encontrar que las mujeres tienen una respuesta más satisfactoria al cierre de las úlceras manejadas por oxígenoterapia, frente a los hombres, llegando a ser esta probabilidad en las medidas de asociación hasta de 2.47 veces. No existen factores fisiológicos vinculados a la condición de género, que determinen mejores respuestas tisulares de la oxidación, en la presencia de altas concentraciones de oxígeno plasmáticas derivadas de la acción de la oxígenoterapia hiperbárica. Por lo tanto, sería tema de otros trabajos encontrar la relación del género, con el cierre de las úlceras arteriales tratadas con oxígenoterapia, teniendo presente otros factores que pueden ocasionar este hecho.
Los resultados del estudio sugieren, que un tratamiento mayor de veinte sesiones de oxígeno hiperbárico es un factor que tendrá mejor relación con el efecto pretendido de cerrar la úlcera. Si se observa a los pacientes que tuvieron esta condición, podemos ver que lograron en la razón de momios, 2.45 veces más probabilidades del cierre de la úlcera que quienes recibieron menos de este número. Aunque en los elementos estadísticos no se hayan encontrado niveles de significancia, se puede señalar también, que la dosificación de las ATA en la instauración de oxígeno hiperbárico para las úlceras arteriales debe estar por encima de 1.5 ATA. Ya que las personas que fueron manejadas con esta prescripción, registraron 2.46 veces más posibilidades de tener el cierre de la úlcera, en comparación a los que estuvieron por debajo del nivel de aplicación de 1.5 ATA.

Finalmente, los resultados de este estudio muestran una evolución favorable de las personas con úlceras arteriales en la resolución de su compromiso, en un tiempo corto superior al que se obtiene en las formas convencionales de tratamiento, sin complicaciones. Por lo consiguiente, el oxígeno hiperbárico muestra situaciones, que darían lugar a su práctica, como una intervención positiva, y segura para el manejo de úlceras arteriales. Ésta debería aplicarse con mayor frecuencia, para reducir las situaciones de morbimortalidad causadas por la presencia de úlceras y reduciendo los impactos clínicos, económicos y sociales que pueden ocasionar, facilitando no solo la mejoría clínica, con medidas menos traumáticas, sino la reincorporación funcional del paciente, en menor tiempo y con mejores condiciones $(1,11-14)$.

\section{REFERENCIAS}

1. Uzun G, Yildiz S, Aktas S. Hyperbaric oxygen therapyin the management of nonhealing wounds in patients with critical limb ischemia. Therapy. 2008; 5(1): 99-108.

2. Szijarto A, Turoczi Z, Aranyi P, Nagy Z, Gyurkovics E. Acute and critical ischemia of the lower limb. Orv Hetil. 2010; 151(50): 2057-2066.

3. Arain FA, Cooper LT Jr. Peripheral arterial disease: diagnosis and management. Mayo Clin Proc. 2008; 83(8): 944-949.

4. Korber A, Klode J, Al-Benna S, Wax C, et al. Etiology of chronic leg ulcers in 31.619 patients in Germany analyzed by an expert survey. J Dtsch Dermatol Ges. 2011; 9(2): 116-121.

5. Korber A, Schadendorf D, Dissemond J. Causes of leg ulcers. Analysis of the data from a dermatologic wound care center. Hautarzt. 2009; 60(6): 483-488.

6. Shigematsu H. Epidemiology from the Trans Atlantic InterSociety consensus group guidelines II. Nippon Geka Gakkai Zasshi. 2007; 108(4): 171-175.

7. Gardner AW, Afag A. Management of lower extremity peripheral arterial disease. Journal of Cardiopulm Rehabil Prev. 2008; 28(6): 349-357. 
8. Met R, Van Lienden KP, Koelemay MJ, Bipat S, et al. Subintimal angioplasty for peripheral arterial occlusive disease: a systematic. Review Cardiovasc Intervent Radiol. 2008; 31(4): 687-697.

9. Gist S, Tio-Matos I, Falzgraf S, Cameron S, Beebe M. Wound Healing in older adults. Aging Healt. 2009; 5 (6): 851-866

10. Bader MS. Diabetic foot infection. Am Fam Physician. 2008; 78(1): 71-79.

11. Stirban A, Lentrodt S, Nandrean S, Pop A et al. Functional changes in microcirculation during hyperbaric and normobaric oxygen therapy. Undersea Hyperb Med. 2009; 36(5): 381-390.

12. Kulikovsky M, Gil T, Mettanes I, Karmeli R et al. Hyperbaric oxygen therapy for non healing wounds. Isr Med Assoc J. 2009; 11(8): 480-485.

13. Melamed Y, Bitterman $H$. Non healing wounds and hyperbaric oxygen: a growing awareness. Isr Med Assoc J. 2009; 11(8): 498-500.
14. Thackham JA, McElwain DL, Long RJ. The use of hyperbaric oxygen therapy to treat chronic wounds: A review. Wound Repair Regen. 2008; 16(3): 321-330.

15. Hernández B, Velasco E. Encuestas transversales. Salud Publica Mex. 2000; 42(5): 447-455.

16. Hernández M, Garrido F, López S. Diseño de estudios epidemiológicos. Salud Publica Mex. 2000; 42(2): 144-154.

17. Benizri E, Ginouves A, Berra $\mathrm{E}$. The magic of the hipoxiasignaling cascade. Cell Mol Life Sci. 2008; 65: 1133-1149.

18. Zelen CM, Stover V, Nielson D, Cunningham M. A prospective study of negative pressure wound therapy with integrated irrigation for the treatment of diabetic foot ulcers Eplasty, Febrero 2011.

19. Uccioli L, Gandini R, Giurato L, Fabiano S et al. Long-term outcomes of diabetic patients with critical limb ischemia followed in a tertiary referral diabetic food clinic. Diabetes Care. 2010; 33(5): 977-982 